\title{
A Dynamic Contagion Process and an Application to Credit Risk
}

\section{Abstract}

We introduce a new point process, the dynamic contagion process, by generalising the Hawkes process and the Cox process with shot noise intensity. Our process includes both self-excited and externally excited jumps, which could be used to model the dynamic contagion impact from endogenous and exogenous factors of the underlying system. We have systematically analysed the theoretical distributional properties of this new process, based on the piecewise deterministic Markov process theory developed by Davis (1984), and the extension of the martingale methodology used by Dassios and Jang (2003). The analytic expressions of the Laplace transform of the intensity process and the probability generating function of the point process have been derived. An explicit example of specified jumps with exponential distributions is also given. The object of this study is to produce a general mathematical framework for modelling the dependence structure of arriving events with dynamic contagion, which has the potential to be applicable to a variety of problems in economics, finance and insurance. We provide an application of this process to credit risk, and the simulation algorithm for further industria

\section{Dynamic Contagion Process}

Mathematical Definition

The dynamic contagion process is a point process $N_{t}$, such that

$P\left\{N_{t+\Delta t}-N_{t}=1 \mid N_{t}\right\}=\lambda_{t} \Delta t+o(\Delta t), \quad P\left\{N_{t+\Delta t}-N_{t}>1 \mid N_{t}\right\}=o(\Delta t)$, where $\Delta t$ is a sufficient small time interval, and the non-negative intensity

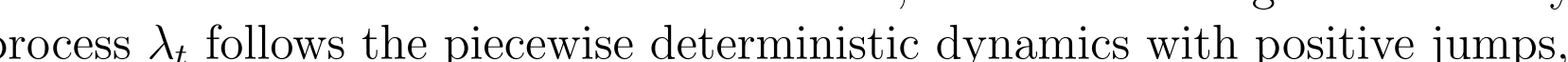
$\lambda_{t}=a+\left(\lambda_{0}-a\right) e^{-\delta t}+\int_{0}^{t} e^{-\delta(t-s)} \mathrm{d} Y_{s}^{(1)}+\int_{0}^{t} e^{-\delta(t-s)} \mathrm{d} Y_{s}^{(2)}$,

where

- $a \geq 0$ is the reversion level;

- $\lambda_{0}>0$ is the initial value of $\lambda_{t}$

- $\delta>0$ is the constant rate of exponential decay

- $Y_{t}^{(1)}=\sum_{i=1}^{M_{t}} Y_{i}^{(1)},\left\{Y_{i}^{(1)}\right\}_{i=1,2 \ldots}$ is a sequence of independent identical distributed positive random variables (externally excited jumps) with distribution function $H(y), y>0$, and $M_{t}$ is a Poisson process with constant intensity $\rho>0$

- $Y_{t}^{(2)}=\sum_{j=1}^{N_{t}} Y_{j}^{(2)},\left\{Y_{j}^{(2)}\right\}_{j=1,2 \ldots .}$ is a sequence of independent identical distributed positive random variables (self excited jumps) with distribution function $G(y), y>0$;

- The sequences $\left\{Y_{i}^{(1)}\right\}_{i=1,2, \ldots},\left\{Y_{j}^{(2)}\right\}_{j=1,2, \ldots}$ and the point process $M_{t}$ are assumed to be independent of each other.

The infinitesimal generator of the dynamic contagion process $\left(\lambda_{t}, N_{t}, t\right)$ acting on a function $f(\lambda, n, t)$ within its domain $\Omega(\mathcal{A})$ is given by

$\mathcal{A} f(\lambda, n, t)=\frac{\partial f}{\partial t}+\delta(a-\lambda) \frac{\partial f}{\partial \lambda}+\rho\left(\int_{0}^{\infty} f(\lambda+y, n, t) \mathrm{d} H(y)-f(\lambda, n, t)\right)$ $+\lambda\left(\int_{0}^{\infty} f(\lambda+y, n+1, t) \mathrm{d} G(y)-f(\lambda, n, t)\right)$

where $\Omega(\mathcal{A})$ is the domain for the generator $\mathcal{A}$ such that $f(\lambda, n, t)$ is differentiable with respect to $\lambda, t$ for all $\lambda, n$ and $t$, and

$$
\begin{gathered}
\left|\int_{0}^{\infty} f(\lambda+y, n, t) \mathrm{d} H(y)-f(\lambda, n, t)\right|<\infty ; \\
\left|\int_{0}^{\infty} f(\lambda+y, n+1, t) \mathrm{d} G(y)-f(\lambda, n, t)\right|<\infty
\end{gathered}
$$

\section{Graphic Illustration}

To give an intuitive picture of this new process, we present the figure below for illustrating how the externally excited jumps $Y^{(1)}$ (marked by single arrow $\downarrow$ and self excited jumps $Y^{(2)}$ (marked by double arrow $\uparrow$ ) in the intensity process $\lambda_{t}$ interact with its dynamic contagion point process $N_{t}$.

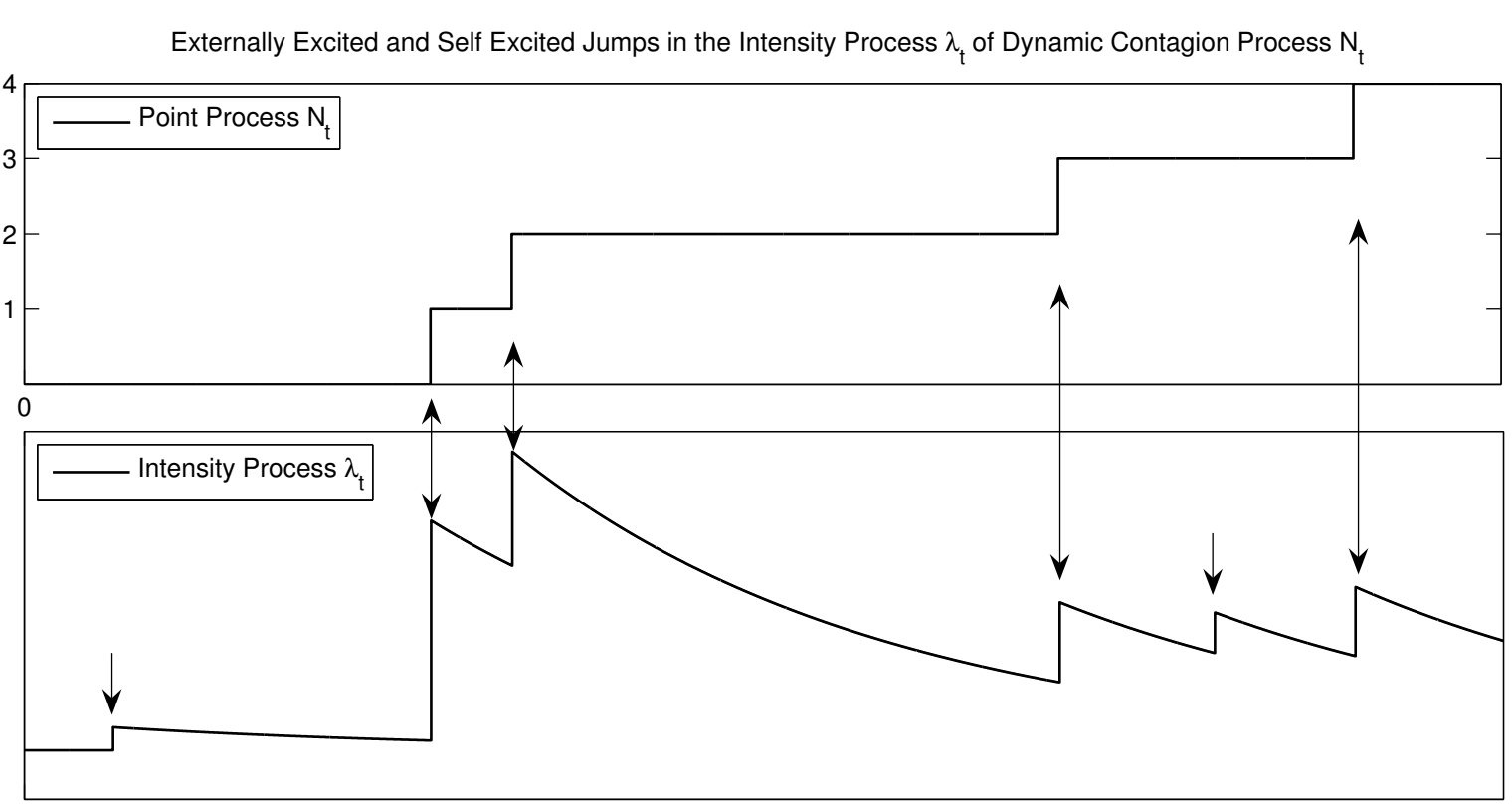

In this more general framework of the dynamic contagion process, the classic Cox process with shot noise intensity, used by Dassios and Jang (2003) for pricing catastrophe reinsurance and derivatives, can be recovered, by setting reversion level $a=0$ and eliminating the self excited jumps $Y^{(2)}$; The Hawkes process (with the exponential decay), used by Errais, Giesecke and Goldberg
(2009) for modelling the portfolio credit risk in a top-down approach framework, can be recovered, by setting the intensity $\rho=0$ of the externally excited jumps $Y^{(1)}$.
Angelos Dassios ${ }^{\dagger}$ and Hongbiao Zhao ${ }^{\ddagger}$

Department of Statistics, London School of Economics

${ }^{\dagger}$ A.Dassios@lse.ac.uk, ${ }^{\ddagger}$ H.Zhao1@lse.ac.uk
Laplace Transform of the Intensity Process $\lambda_{t}$

The conditional Laplace transform $\lambda_{T}$ given $\lambda_{0}$ at time $t=0$, under the condition $\delta>\mu_{1_{G}}$, is given by

$\mathbb{E}\left[e^{-v \lambda_{T}} \mid \lambda_{0}\right]=\exp \left(-\int_{\mathcal{G}_{v, 1}^{-1}(T)}^{v} \frac{a \delta u+\rho[1-\hat{h}(u)]}{\delta u+\hat{g}(u)-1} \mathrm{~d} u\right) \times \exp \left(-\mathcal{G}_{v, 1}^{-1}(T) \lambda_{0}\right)$,

$$
\begin{gathered}
\mu_{1_{G}}=: \int_{0}^{\infty} y \mathrm{~d} G(y), \quad \mathcal{G}_{v, 1}(L)=: \int_{L}^{v} \frac{\mathrm{d} u}{\delta u+\hat{g}(u)-1} \\
\hat{h}(u)=: \int_{0}^{\infty} e^{-u y} \mathrm{~d} H(y), \quad \hat{g}(u)=: \int_{0}^{\infty} e^{-u y} \mathrm{~d} G(y) .
\end{gathered}
$$

The Laplace transform of the asymptotic distribution of $\lambda_{T}$ is given by

$$
\lim _{T \rightarrow \infty} \mathbb{E}\left[e^{-v \lambda_{T}} \mid \lambda_{0}\right]=\exp \left(-\int_{0}^{v} \frac{a \delta u+\rho[1-\hat{h}(u)]}{\delta u+\hat{g}(u)-1} \mathrm{~d} u\right),
$$

and this is also the Laplace transform of the stationary distribution of $\left\{\lambda_{t}\right\}_{t \geq 0}$.

Example: Jumps with Exponential Distributions

If both the externally excited and self excited jumps follow exponential distributions, i.e. $Y^{(1)} \sim \operatorname{Exp}(\alpha)$ and $Y^{(2)} \sim \operatorname{Exp}(\beta)$, then, under the stationarity condition $\delta \beta>1$, the stationary distribution of $\left\{\lambda_{t}\right\}_{t \geq 0}$ is given by

$$
\begin{cases}a+\tilde{\Gamma}_{1}+\tilde{\Gamma}_{2} & \text { for } \alpha \geq \beta \\ a+\tilde{\Gamma}_{3}+\tilde{B} & \text { for } \alpha<\beta \text { and } \alpha \neq \beta-\frac{1}{\delta} \\ a+\tilde{\Gamma}_{4}+\tilde{P} & \text { for } \alpha=\beta-\frac{1}{\delta}\end{cases}
$$

where independent random variables

$\tilde{\Gamma}_{1} \sim \operatorname{Gamma}\left(\frac{1}{\delta}\left(a+\frac{\rho}{\delta(\alpha-\beta)+1}\right), \frac{\delta \beta-1}{\delta}\right) ; \tilde{\Gamma}_{2} \sim \operatorname{Gamma}\left(\frac{\rho(\alpha-\beta)}{\delta(\alpha-\beta)+1}, \alpha\right)$;

$\tilde{\Gamma}_{3} \sim \operatorname{Gamma}\left(\frac{a+\rho}{\delta}, \frac{\delta \beta-1}{\delta}\right) ; \tilde{B} \underline{\underline{\mathcal{D}}} \sum_{i=1}^{N_{1}} X_{i}^{(1)}, N_{1} \sim \operatorname{NegBin}\left(\frac{\rho}{\delta} \frac{\beta-\alpha}{\gamma_{1}-\gamma_{2}}, \frac{\gamma_{2}}{\gamma_{1}}\right), X_{i}^{(1)} \sim$

$\tilde{\Gamma}_{4} \sim \operatorname{Gamma}\left(\frac{a+\rho}{\delta}, \alpha\right) ; \tilde{P} \stackrel{\mathcal{D}}{=} \sum_{i=1}^{N_{2}} X_{i}^{(2)}, N_{2} \sim \operatorname{Poisson}\left(\frac{\rho}{\delta^{2} \alpha}\right), X_{i}^{(2)} \sim \operatorname{Exp}(\alpha) ;$

and $\gamma_{1}=\max \left\{\alpha, \frac{\delta \beta-1}{\delta}\right\}, \gamma_{2}=\min \left\{\alpha, \frac{\delta \beta-1}{\delta}\right\} . \quad \tilde{B}$ follows a compound negative binomial distribution with underlying exponential jumps; $\tilde{P}$ follows a compound Poisson distribution with underlying exponential jumps.

Particularly, for the non-self-excited case, $\lambda_{T}$ follows a shifted Gamma distribution,

$$
\begin{gathered}
\lambda_{T} \stackrel{\underline{D}}{=} a+\tilde{\Gamma}_{5}, \\
\tilde{\Gamma}_{5} \sim \operatorname{Gamma}\left(\frac{\rho}{\delta}, \alpha\right),
\end{gathered}
$$

which recovers the result by Dassios and Jang (2003); For the Hawkes process, $\lambda_{T}$ follows a shifted Gamma distribution,

$$
\lambda_{T} \stackrel{\mathcal{D}}{=} a+\tilde{\Gamma}_{6},
$$

where

$$
\tilde{\Gamma}_{6} \sim \operatorname{Gamma}\left(\frac{a}{\delta}, \frac{\delta \beta-1}{\delta}\right)
$$

Probability Generating Function of the Point Process $N_{t}$ The conditional probability generating function of $N_{T}$ given $\lambda_{0}$ and $N_{0}=0$ at time $t=0$, under the condition $\delta>\mu_{1_{G}}$, is given by

$$
\begin{aligned}
& \qquad \mathbb{E}\left[\theta^{N_{T}} \mid \lambda_{0}\right]=\exp \left(-\int_{0}^{\mathcal{G}_{0, \theta}^{-1}(T)} \frac{a \delta u+\rho[1-\hat{h}(u)]}{1-\delta u-\theta \hat{g}(u)} \mathrm{d} u\right) \times \exp \left(-\mathcal{G}_{0, \theta}^{-1}(T) \lambda_{0}\right), \\
& \text { where } \\
& \qquad \mathcal{G}_{0, \theta}(L)=: \int_{0}^{L} \frac{\mathrm{d} u}{1-\delta u-\theta \hat{g}(u)} \quad 0 \leq \theta<1 .
\end{aligned}
$$

\section{An Application in Credit Risk}

Our motivation of applying the dynamic contagion process to model the credit risk is a combination of Duffie and Singleton (1999) and Lando (1998). Duffie and Singleton (1999) introduced the affine processes to model the default intensity. Lando (1998), the extension of Jarrow, Lando and Turnbull (1997), used the state of credit ratings as an indicator of the likelihood of default, and modelled the underlying credit rating migration driven by a probability transition matrix with Cox processes in a finite-state Markov process framework. However, we go beyond this and model the bad events that can possibly lead to credit default, and the number and the intensity of these events are modelled by the dynamic contagion process.

The point process $N_{t}$ is to model the number of bad events released from the thderlying company. It is driven by a series of bad events $Y$ (2) from itsel process $\lambda_{\text {. The }}$ The impect of each event decays expenentially with cos int $\delta$. We assume each jump, or bad event, can result to default with a constant probability $d, 0<d \leq 1$, which measures and quantifies the resistance level. Therefore, the survival probability conditional on the (initial) current intensity $\lambda_{0}$ at time $T$ is $P(T)=\mathbb{E}\left[(1-d)^{N_{T}} \mid \lambda_{0}\right]$, which can be calculated simply sity $\lambda_{0}$ at time $T$ is $P_{s}(T)=\mathbb{E}\left[(1-d)^{N_{T}} \mid \lambda_{0}\right]$, which can be calculated simply
by letting $\theta=1-d$ in the conditional probability generating function. By setting the parameters $\left(a, \rho, \delta ; \alpha, \beta ; \lambda_{0}\right)=(0.7,0,5,2 ; 20,1,5 ; 0.7)$, the term structure of the survival probabilities $p_{s}(T)$ based on $d=2 \%, 10 \%, 20 \%$ and $100 \%$ are shown in the figure below.

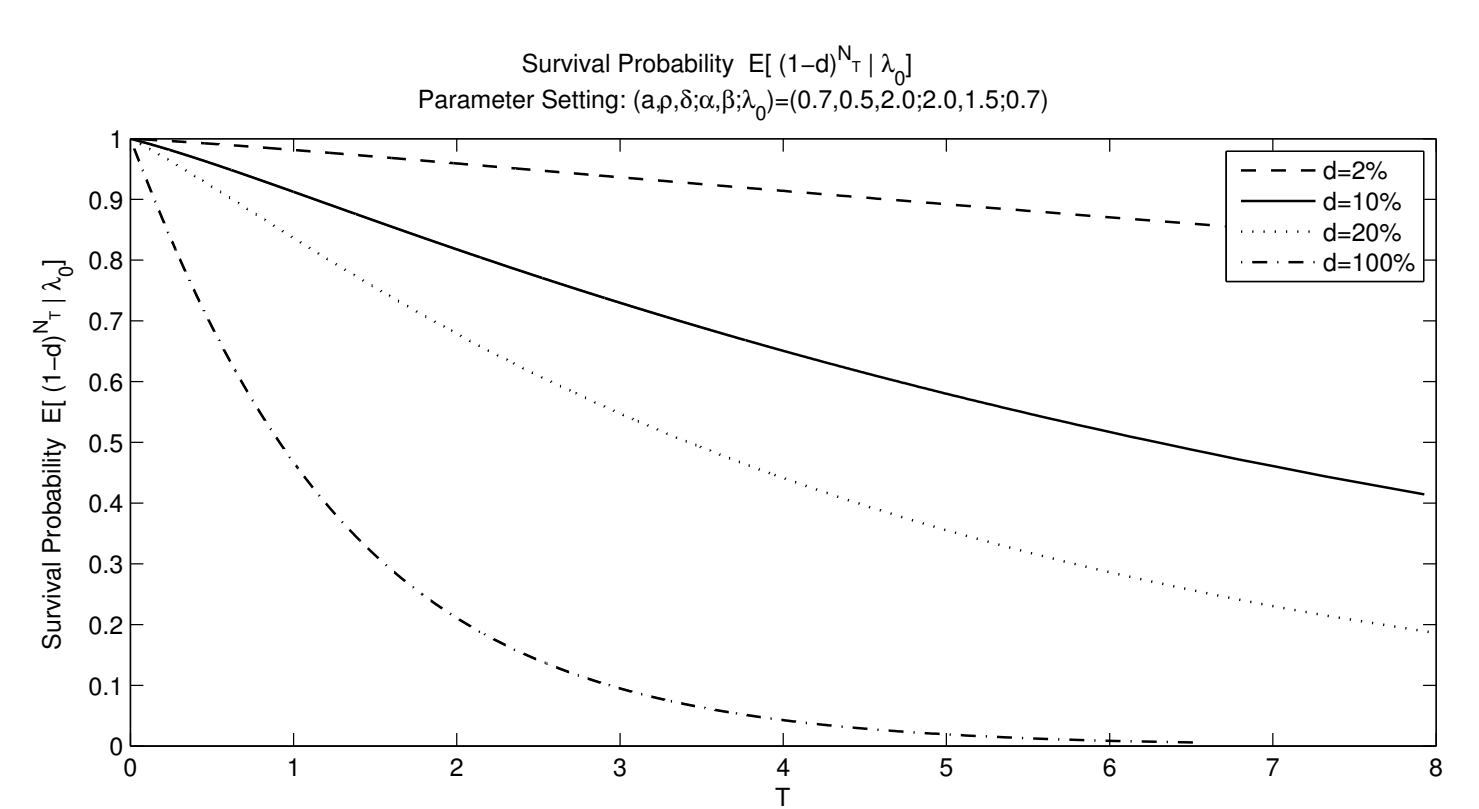

By contrast with Lando (1998), we possibly could consider different values of $d$ correspond to different credit ratings, by assuming these bad events are all related to the company's credit ratings.

We also provide a comparison for the survival probabilities based on three main processes discussed in this paper: dynamic contagion process, Hawkes process (by setting $\rho=0$ ) and non-self-exciting process (by setting $\beta=\infty$ ), with the same parameter setting and fixed $d=10 \%$. The results are shown in the figure below.

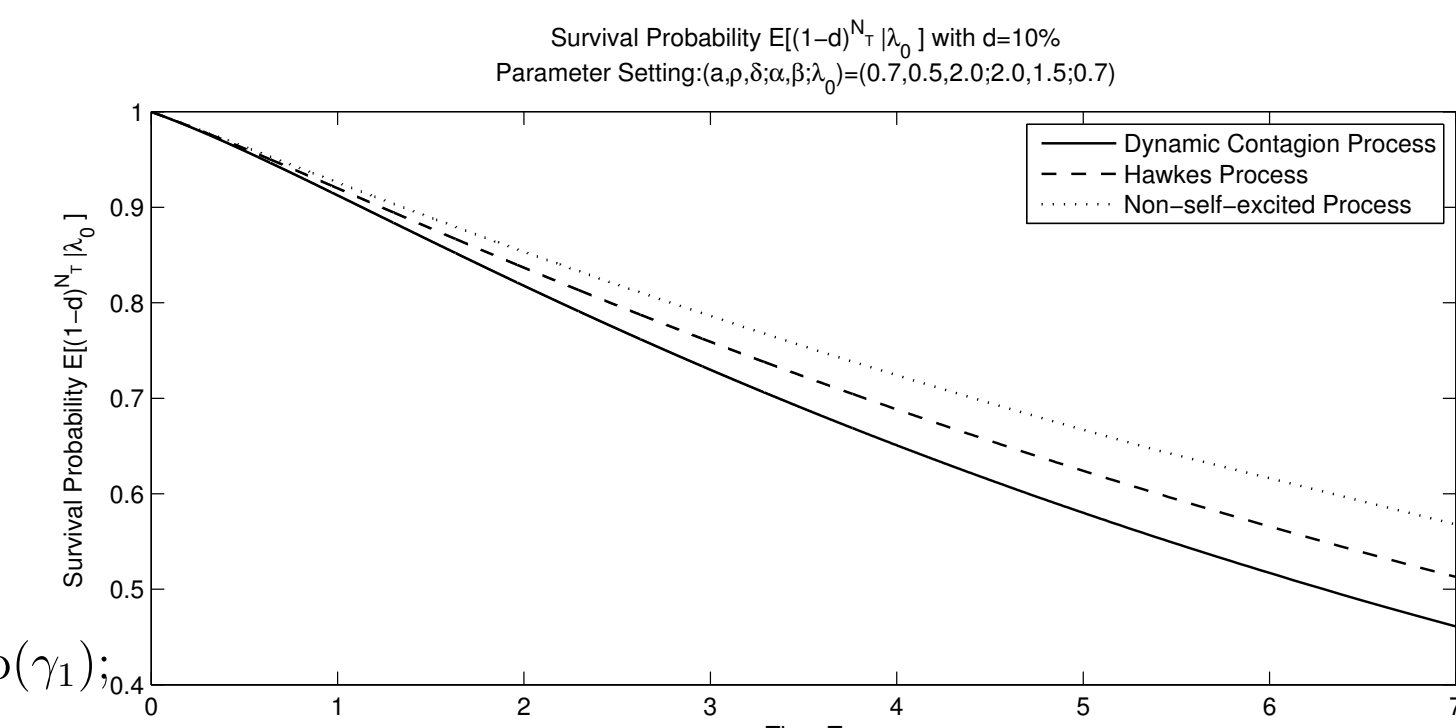

We can see that, the dynamic contagion process, as the most general case of the three processes, generates the lowest survival probability, and the differand exetrons axpere the economic downturn involving more clustering bad economic events.

\section{Monte Carlo Simulation}

To make easier for further industrial applications and statistical analysis, alternatively, we derive the simulation algorithm for one sample path of the general dynamic contagion process $\left(N_{t}, \lambda_{t}\right)$, which applies to any distribution assumption for jump sizes, $H(y)$ and $G(y)$ for externally and self excited jumps, respectively. Here, we use the same parameter setting under the exponential distribution assumption for the jump sizes, and for instance one
simulated sample path $\left(N_{t}, \lambda_{t}\right)$ with $T=50$ is provided in the figure below. For comparison, the theoretical expectations $\mathbb{E}\left[\lambda_{t}\right], \mathbb{E}\left[\lambda_{t} \mid \lambda_{0}\right]$ and $\mathbb{E}\left[N_{t}\right]$ are also plotted

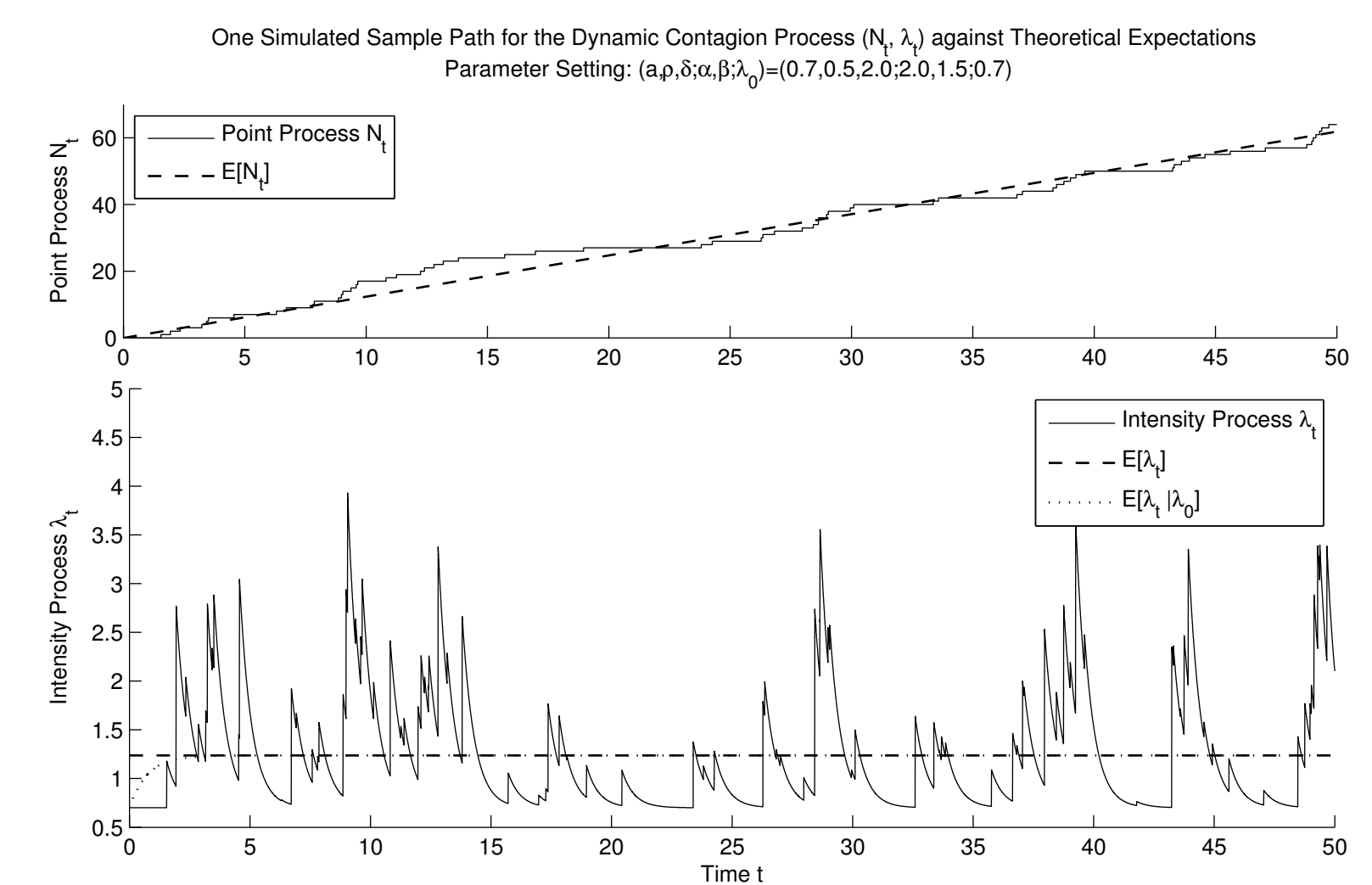

References

Dassios, A., Embrechts, P.: Martingales and Insurance Risk. Communications in Statistics-Stochastic Models 5(2): 181-217 (1989)

Dassios, A., Jang, J.: Pricing of Catastrophe Reinsurance and Derivatives Using the Cox Process with Shot Noise Intensity. Finance 83 Stochastics 7(1)

Dassios, A., Zhao, H.: A Dynamic Contagion Process. Advances in Applied robability 43(3) (2011)

Pris, M.H.A.. Piecewise Deterministic Markov Processes: A General Class Journal of the Royal Statistical Society B

Duffie, D., Filipović, D. Schachermayer, W.: Affine Processes and Applica(2003) Spectra of Some Self-exciting and Mutually Exciting Point Processes. Biometrika 58(1), 83-90 (1971) 